\title{
Calibration of Infrared Cameras with Microbolometers
}

\author{
Helmut Budzier, Gerald Gerlach \\ Technische Universität Dresden, Faculty of Electro Technology and Information Technology, \\ Institute of Solid-state Electronics, \\ Helmut.budzier@tu-dresden.de
}

\begin{abstract}
A description will be given of the calibration process for accurate measurement of temperature in uncooled infrared cameras on the basis of microbolometer arrays. Calibration is used to calculate a temperature-proportional output signal (IR image) from the measurement signal (raw image) taking into account all technical and physical properties of the IR camera. The individual steps of the calibration are shown by way of example. After commissioning of the IR camera first all defective pixels are detected. Then the balance of the pixel characteristic curves (correction of the nonuniformity) is carried out using two-point correction. In microbolometers that are not temperaturestabilized, the dependence of the sensor parameters of the temperature sensor must also be considered. Ambient temperature changes are compensated for by shutter correction. The final stage involves radiometric calibration, which establishes the relationship between pixel voltage and target object temperature.
\end{abstract}

Key words: Infrared, uncooled cameras, calibration, microbolometer

\section{Introduction}

In recent years, thermography has made dramatic developments. Enabling this huge market success are infrared (IR) image sensors based on microbolometer arrays, which have excellent infrared thermal and spatial resolution. Microbolometers operate at room temperature and do not require expensive cooling /1/. They therefore have low power consumption and allow a compact camera design. The principle of an uncooled IR camera is shown in Figure 1. Both the major system components, i.e. the IR optics, the microbolometer camera electronics and also the calibration of the camera, are of particular importance for gaining a greater measurement accuracy /2/.

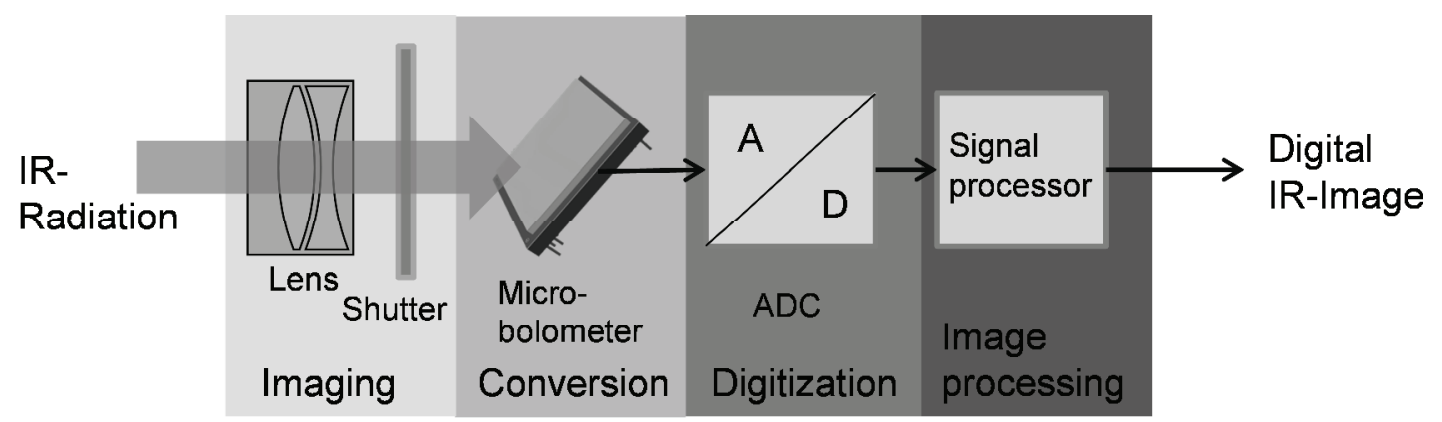

Figure 1: Signal flow of an uncooled IR camera

Due to the complicated manufacturing process, the individual pixels of a microbolometer array have different characteristics. The calibration process eliminates these differences and determines the relationship between the signal voltage of a pixel and the temperature of the object to be measured.

\section{Calibration}

A radiometer measures the radiation flux of the target object and generates an output signal which, as a result of the calibration, is proportional to the temperature of the object
/3/. A radiometric IR camera displays the true temperature of an object as accurately as possible. During calibration, a black body radiator is always used as an object. The calibration is used here to calculate a temperature-proportional output signal IR image from the measurement signal (raw), taking into account all technical and physical properties of the IR camera. The steps necessary for this are summarized in Figure 2. Finally, the individual stages will be presented with the help of an example. 

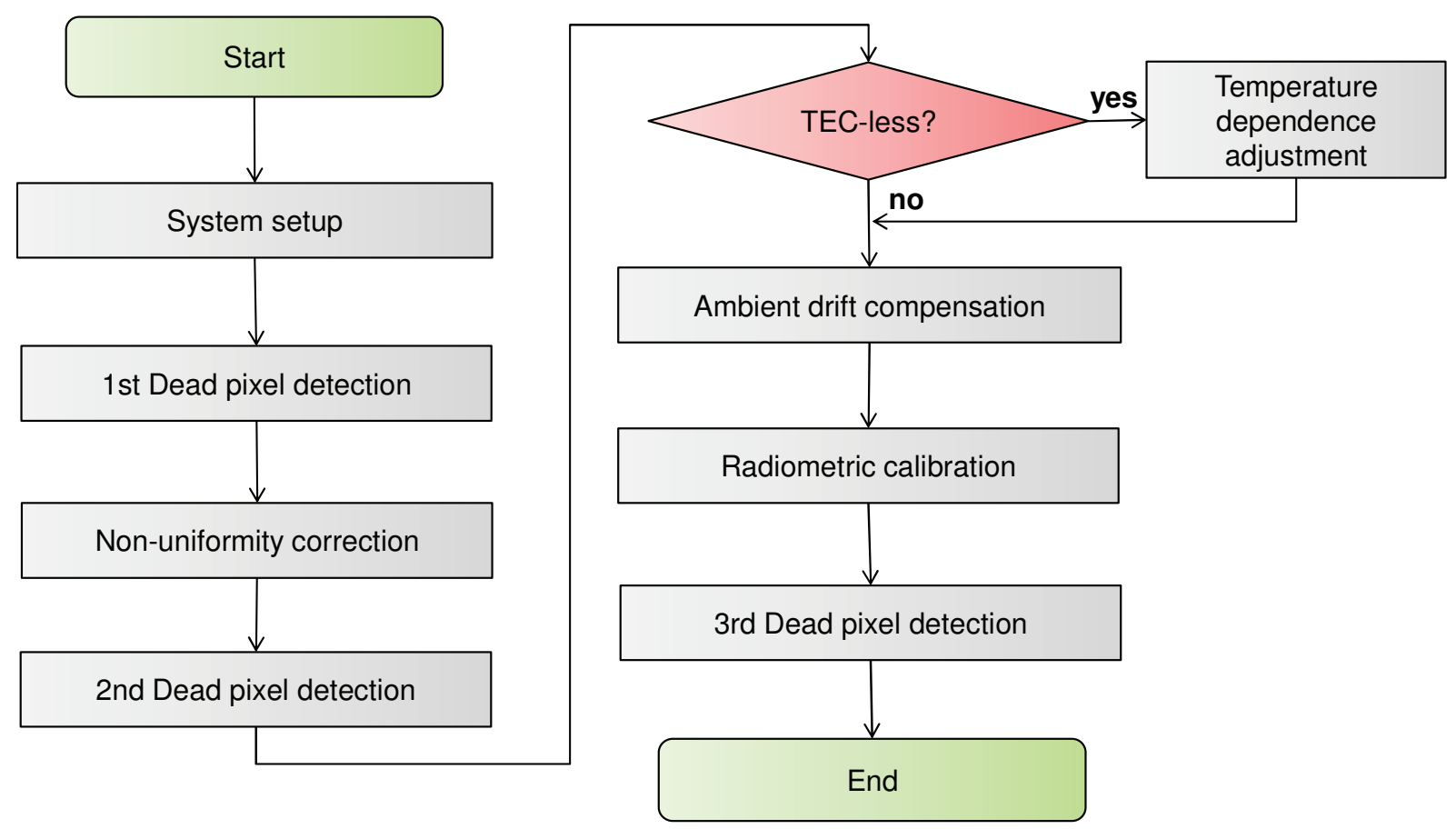

Figure 2: Sequence of calibration

\subsection{Commissioning and correction of defective pixels}

During the commissioning of the IR camera the start-up settings of the operating points of the micrbolometer are carried out. This procedure depends heavily on the microbolometer used and corresponds to the example provided by the manufacturer's procedure. The operating point of the microbolometer is to be chosen so that the desired temperature range of the IR

a)

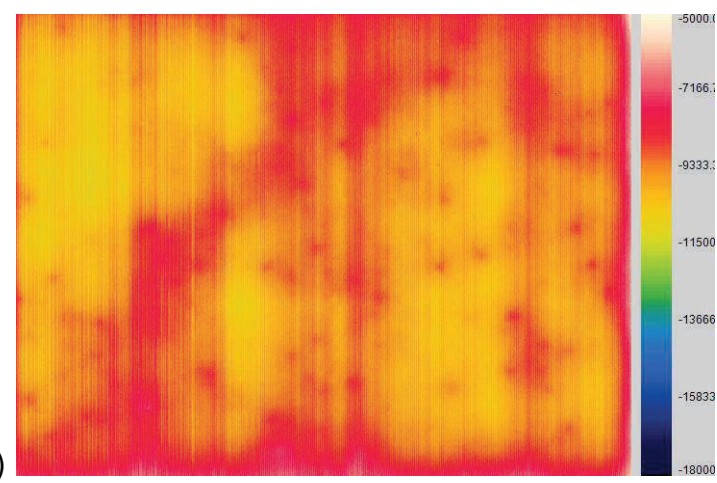

b)

camera can be evaluated. Figure 3 shows the raw image of a microbolometer.

Some pixels do not respond to incident IR radiation or have a distinctly different voltage object temperature characteristic. These defective pixels ("dead" pixels) will be determined step by step and will be substituted by neighbouring pixels using methods of image processing, e.g. with media operators.

b)



Fure 3: Raw image of a microbolometer array. a) IR image with colour bar chart in grey values and b) histogram of raw image; Dynamic range from -32000 to 32000 grey values.

\subsection{Correction of non-uniformity}

All pixels have different DC operating points (offset) and sensitivities. With the help of twopoint correction the IR image is "smoothed out". For this purpose, an average characteristic line, the so-called standard characteristic curve is calculated, around which each pixel characteristic is located. For each pixel with the signal voltage $V_{m n}$, a pair of coefficients offset $t_{m n}$ and gain ${ }_{m n}$ is determined, so that

$$
V_{\text {korr }, m n}=\left(V_{m n}-\text { offset }_{m n}\right) * \text { gain }_{m n}+V_{\text {normoffset }}
$$

applies (Figure 4). Next, the pixel curve is shifted in parallel $\left(-\right.$ offset $\left._{m n}\right)$ and then the 
increase is corrected (* ${ }^{*}$ gain $\left._{m n}\right)$. In conclusion, a constant voltage value can be added to all pixels. The standard curve is thus pushed back $\left(+V_{\text {normoffset }}\right)$. This last step is not necessary in every case, but guarantees that the corrected pixel voltages are in the same range of values as the measured pixel values. This is important, for example, if the correction is implemented in hardware (16-bit fixed-point arithmetic) and the range is limited. In order to reduce the calculation during real-time correction, Eq. (1)

$V_{\text {korr }, m n}=V_{m n} *$ gain $_{m n}+$ offset $_{m n}^{*}$

can be simplified to:

$$
\text { offset }_{m n}^{*}=- \text { offset }_{m n} * \text { gain }_{m n}+V_{\text {normoffset }}
$$

Figure 5 shows the histograms of the coefficients, which are calculated from the raw images.
As a consequence of various measurement deviations interferences, in particular by the temporal noise of the pixel voltage, the determination of the coefficients is faulty. Because of this, a small deviation between the pixels remains in the corrected image. This deviation can be interpreted as spatial noise. To be able to assess the quality of the correction, the characteristic corrigibility $C$ in /4/ is proposed:

$$
C=\sqrt{\frac{V_{S N}^{2}}{V_{T N}^{2}}}
$$

where the noise voltages $V_{T N}$ for the temporal noise and $V_{S N}$ for the spatial noise are used. The corrigibility $C$ is ideal for correcting the characteristics of zero. It is equal to one if the spatial noise has the same value as the temporal noise. If the corrigibility is greater than one, the spatial noise dominates and the non-uniformity is clearly visible in the IR image.



Figure 4: Principle of two-point correction (see text for explanation)

a)



Figure 5: Calculated values of two point correction. of the gain values

\subsection{Temperature dependence correction}

If the temperature of the microbolometer is not constant, as is the case for TEC-less b)

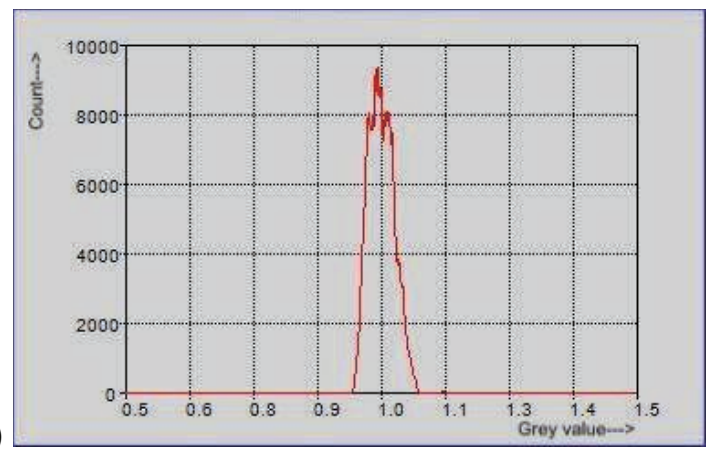

microbolometers, then the sensor sensitivity and offset parameters change with temperature. The dependence of the offset and the sensitivity of a microbolometer array 
cannot be derived from the physical properties of a bolometer resistance. This can only happen with the knowledge of the internal signal processing function. Since the internal signal processing of a microbolometer array is usually not known in detail because of inhouse security measures, the array must be regarded as a black box. In general, the following polynomials can be assumed for the temperature dependence of the offset $O_{V}$ and sensitivity $G_{V}$ of the sensor temperature $\vartheta_{S}$ :

$O_{V}\left(\vartheta_{S}\right)=o_{3} \vartheta_{S}^{3}+o_{2} \vartheta_{S}^{2}+o_{1} \vartheta_{S}+o_{0}$



a)

Figure 6: Temperature dependence of a) the offset values and b) the sensitivity of a TEC-less microbolometer array.

\subsection{Shutter correction}

If the ambient temperature of the IR camera varies, then the inner temperature of the camera also changes. Using the shutter characteristic curve enables the influence of the internal camera temperature $\vartheta_{S}$ on the pixel signal to be determined and corrected. The shutter characteristic curve represents the ratio of the shutter image to the IR image with the shutter open at constant target object temperature, but the changing ambient temperature is: and

$G_{V}\left(\vartheta_{C}\right)=g_{2} \vartheta_{S}^{2}+g_{1} \vartheta_{S}+g_{0}$.

For each camera the polynomial coefficients $O_{0,1,2,3}$ and $g_{0,1,2}$ have to be determined in a complicated measuring regime. However, they are not pixel-specific. In Figure 6 examples are given. The following then applies:

$V_{\text {korr }, m n}=\left[V_{m n}-\right.$ offset $\left._{m n}\right] *$ gain $_{m n} / G_{V}\left(\vartheta_{S}\right)-O_{V}\left(\vartheta_{S}\right)$.

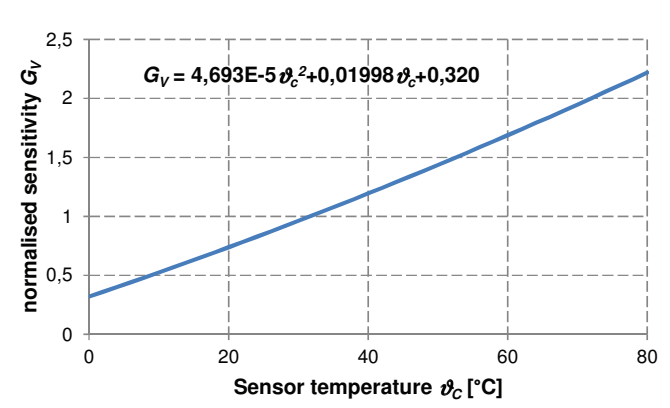

b)

$$
V_{\text {open }}\left(\vartheta_{C}\right)=a_{S h} V_{S h}\left(\vartheta_{C}\right)+V_{O}
$$

with the shutter signal $V_{S h}$, the IR image with open shutter $V_{\text {open }}$, the object voltage $V_{O}$ and the increase ash (Figure 7). Now the signal voltage of the object can be calculated using the shutter image regardless of the ambient temperature or camera temperature:

$$
V_{o}=V_{o}\left(\vartheta_{C}\right)-a_{S h} V_{S h}\left(\vartheta_{C}\right) \text {. }
$$

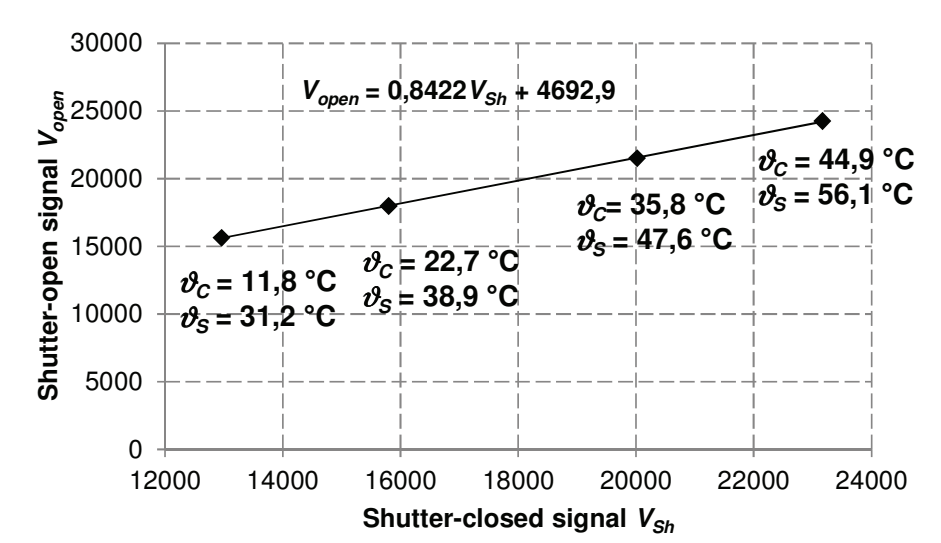

Figure 7: Shutter characteristic curve. Parameters: camera temperature $\vartheta_{C}$ and sensor temperature $\vartheta_{S}$.

\subsection{Radiometric calibration}

Finally, the radiometric calibration calculates the temperature of the measured object from means of a voltage object temperature curve 
According to /4/ it is possible to approximate the sensor output signal with a Planck curve:

$$
V_{D}=\frac{R}{e^{\frac{B}{T_{O}}}-F}+O \text {, }
$$

in which $B, F, O$ and $R$ are the regression coefficients to be determined. Then the inverse function is applied to calculate the object temperature $T_{O}$ for Eq. (10):

$$
T_{O}=\frac{B}{\ln \left(\frac{R}{V_{D}-O}+F\right)} .
$$

The coefficients allow a physical interpretation. The value $O$ is a general offset. Using this, the

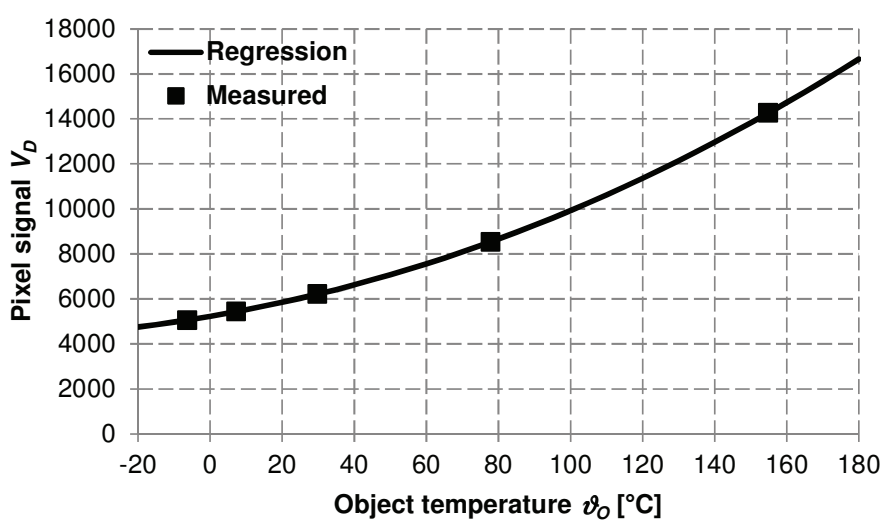

Figure 8: Pixel voltage object curve with $B=1501.4 \mathrm{~K} ; F=1.946 ; R=326507$ and $O=3881$.

\section{Summary}

The calibration of an uncooled IR camera is a complex and lengthy process, which significantly affects its cost. The calibration described allows the measurement of absolute temperatures with a measurement uncertainty characteristic curve can be shifted in parallel in the $y$ direction. The coefficient $R$ represents the system response of the IR camera and is the counterpart to the system sensitivity $R_{V}$. For the coefficient $B$ Planck's radiation law:

$$
B=\frac{c_{2}}{\lambda_{B}}
$$

applies.

It thus describes the spectral behavior of the system. The wavelength $\lambda_{B}$ is the effective wavelength of the IR camera. The coefficient $F$ allows an alignment of the non-linearity of the system. An example is shown in figure 8.

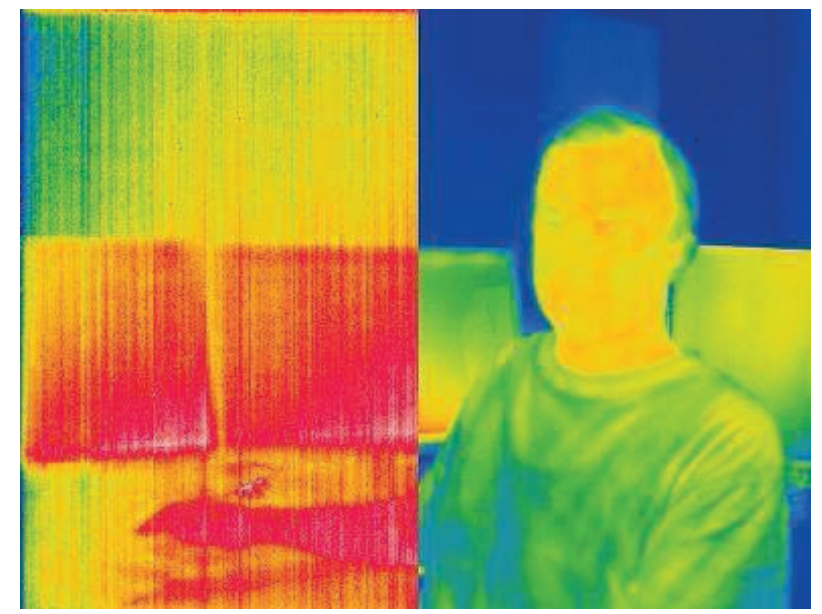

Figure 9: Comparison of a raw image (left half of picture) with a calibrated image (right half of picture). 


\section{Literature}

/1/ Budzier, H.; Gerlach, G.: Thermal Infrared Sensor. John Wiley \& Sons, Chichester, 2011.

/2/ Budzier, H.: Radiometrische Kalibrierung ungekühlter Infrarotkameras. TUDpress, Dresden, 2014.

/3/ DeWitt, D.P., Nutter, G.D.: Theory and Practice of Radiation Thermometry. Wiley, New York, 1989.

/4/ Horny, N.: FPA camera standardization. Infrared Physics \& Technology 44; 2003. 